\title{
Breakdown of the resistor model of CPP-GMR in magnetic multilayered nanostructures
}

\author{
S. Sanvito*, \\ School of Physics and Chemistry, Lancaster University, Lancaster, LA1 $4 Y B$, UK and \\ DERA, Electronics Sector, Malvern, Worcs. WR14 3PS UK \\ C.J. Lambert ${ }^{\dagger}$, \\ School of Physics and Chemistry, Lancaster University, Lancaster, LA1 $4 Y B$, UK \\ J.H. Jefferson \\ DERA, Electronics Sector, Malvern, Worcs. WR14 3PS, UK
}

(March 13, 2018)

\begin{abstract}
We study the effect on CPP GMR of changing the order of the layers in a multilayer. Using a tight-binding simple cubic two band model $(s-d)$, magneto-transport properties are calculated in the zero-temperature, zero-bias limit, within the Landauer-Büttiker formalism. We demonstrate that for layers of different thicknesses formed from a single magnetic metal and multilayers formed from two magnetic metals, the GMR ratio and its dependence on disorder is sensitive to the order of the layers. This effect disappears in the limit of large disorder, where the results of the widely-used Boltzmann approach to transport are restored.
\end{abstract}

\section{PACS: 73.23-b, 75.70-i, 75.70Pa}

Giant magnetoresistance (GMR) in transition metal magnetic multilayers [1, 2] is a spin filtering effect which arises when the magnetizations of adjacent layers switch from an anti-parallel (AP) to a parallel (P) alignment. The resistance in the anti-aligned state is typically higher than the resistance with parallel alignment, the difference being as large as $100 \%$. This sensitive coupling between magnetism and transport allows the development of magnetic field sensors with sensitivity far beyond that of conventional anisotropic magnetoresistance (AMR) devices. In the most common experimental setup, the current flows in the plane of the layers (CIP), and the resistance is measured with conventional multi-probe techniques. Measurements in which the current flows perpendicular to the planes (CPP) are more delicate because of the small resistances involved. Despite these difficulties the use of superconducting contacts [3], sophisticated lithographic techniques [4], and electrodeposition [5 7], makes such measurements possible (for recent reviews see references [8.9).

A widely adopted theoretical approach to GMR is based on the semi-classical Boltzmann equation within the relaxation time approximation. This model has been developed by Valert and Fert 10,11, and has the great advantage that the same formalism describes both CIP and CPP experiments. In the limit that the spin diffusion length $l_{\mathrm{sf}}$ is much larger than the layer thicknesses (ie in the infinite spin diffusion length limit), this model reduces to a classical two current resistor network, with additional possibly spin-dependent scattering at the interfaces [12]. Despite the undoubted success of this description recent experiments [13,14] have drawn attention to the possibility of new features which lie outside the theory. Two important and central predictions of this model are that the CPP GMR ratio is independent of the number of bilayers in the case that the total multilayer length is not constrained to be constant, and furthermore is independent of the order of the magnetic layers in the case of different magnetic species. An apparent violation of the first prediction has been observed in CIP and $\mathrm{CPP}$ measurements 15,13, and of the second prediction in CPP measurements 13.14. However a convincing theoretical explanation is lacking.

The aim of this letter is to provide a quantitative description of the breakdown of the resistor model in diffusive CPP multilayers in the limit of infinite spinrelaxation length. To illustrate this breakdown, consider a multilayer consisting of two independent building blocks, namely a $(\mathrm{N} / \mathrm{M})$ and a $\left(\mathrm{N} / \mathrm{M}^{\prime}\right)$ bilayer, where $\mathrm{M}$ and $\mathrm{M}^{\prime}$ represent magnetic layers of different materials or of the same material but with different thicknesses and $\mathrm{N}$ represents normal metal 'spacer' layers. From an experimental point of view $\mathrm{M}$ and $\mathrm{M}^{\prime}$ must possess different coercive fields, in order to allow AP alignment. In the case of 14] this is achieved by considering respectively Co and $\mathrm{Ni}_{84} \mathrm{Fe}_{16}$ layers with $\mathrm{Ag}$ as non-magnetic spacer, while in 113] both the layers are $\mathrm{Co}$ (with $\mathrm{Cu}$ as spacer) but with

*e-mail: sanvito@dera.gov.uk

$\dagger$ e-mail:c.lambert@lancaster.ac.uk 
different thicknesses (respectively $1 \mathrm{~nm}$ and $6 \mathrm{~nm}$ ). Two kinds of multilayer can be deposited. The first, that we call type $\mathrm{I}$, consists of a $\left(\mathrm{N} / \mathrm{M} / \mathrm{N} / \mathrm{M}^{\prime}\right) \times \mu$ sequence where the species $\mathrm{M}$ and $\mathrm{M}^{\prime}$ are separated by an $\mathrm{N}$ layer and the group of four layers is repeated $\mu$ times. The second, that we call type II, consists of a $(\mathrm{N} / \mathrm{M}) \times \mu\left(\mathrm{N} / \mathrm{M}^{\prime}\right) \times \mu$ sequence, where the multilayers $(\mathrm{N} / \mathrm{M}) \times \mu$ and $\left(\mathrm{N} / \mathrm{M}^{\prime}\right) \times \mu$ are arranged in series. If the coercive fields of $\mathrm{M}\left(H_{M}\right)$ and $\mathrm{M}^{\prime}\left(H_{M^{\prime}}\right)$ are different $\left(\mathrm{eg} H_{M}<H_{M^{\prime}}\right)$ and if $\mathrm{N}$ is long enough to decouple adjacent magnetic layers, the AP configuration can be achieved in both type I and type II multilayers by applying a magnetic field $H$ whose intensity is $H_{M}<H<H_{M^{\prime}}$. The $\mathrm{AP}$ configuration is topologically different in the two cases, because in type I multilayers it consists of AP alignment of adjacent magnetic layers (conventional AP alignment), while in type II multilayers it consists of the AP alignment between the $(\mathrm{N} / \mathrm{M}) \times \mu$ and $\left(\mathrm{N} / \mathrm{M}^{\prime}\right) \times \mu$ portions of the multilayer, within which the alignment is parallel (see figure $1 \mathrm{a}$ a and figure 1 $\mathrm{b}$ ). From the point of view of a resistor network description of transport, the two configurations are equivalent, because they possess the same number of magnetic and non-magnetic layers, and the same number of $\mathrm{N} / \mathrm{M}$ and $\mathrm{N} / \mathrm{M}^{\prime}$ interfaces. Hence the GMR ratio must be the same. In contrast the GMR ratio of type I multilayers is found experimentally to be larger than that of type II multilayers [13, 14 , and the difference between the two GMR ratios increases with the number of bilayers. In the case of [13] the GMR ratio of both type I and type II multilayers increases with the number of bilayers, which again lies outside the resistor network model.

In this Letter we demonstrate for the first time that a description which incorporates phase-coherent transport over long length scales can account for such experiments. To illustrate this we have simulated type I and type II multilayers using a $\mathrm{Co} / \mathrm{Cu}$ system with different thicknesses for the Co layers, namely $t_{\mathrm{Cu}}=10 \mathrm{AP}$, $t_{\mathrm{Co}}=10 \mathrm{AP}, t_{\mathrm{Co}}^{\prime}=40 \mathrm{AP}$. The technique for computing transport properties is based on a three dimensional simple cubic tight-binding model with nearest neighbor couplings and two degrees of freedom per atomic site. The general spin-dependent Hamiltonian is

$$
H^{\sigma}=\sum_{i, \alpha} \epsilon_{i}^{\alpha \sigma} c_{\alpha i}^{\sigma \dagger} c_{\alpha i}^{\sigma}+\sum_{i, j, \alpha \beta} \gamma_{i j}^{\alpha \beta \sigma} c_{\beta j}^{\sigma \dagger} c_{\alpha i}^{\sigma},
$$

where $\alpha$ and $\beta$ label the two orbitals (which for convenience we call $s$ and $d), i, j$ denote the atomic sites and $\sigma$ the spin. $\epsilon_{i}^{\alpha \sigma}$ is the on-site energy which can be written as $\epsilon_{i}^{\alpha}=\epsilon_{0}^{\alpha}+\sigma h \delta_{\alpha d}$ with $h$ the exchange energy and $\sigma=-1$ $(\sigma=+1)$ for majority (minority) spins. In equation (1), $\gamma_{i j}^{\alpha \beta \sigma}=\gamma_{i j}^{\alpha \beta}$ is the hopping between the orbitals $\alpha$ and $\beta$ at sites $i$ and $j$, and $c_{\alpha i}^{\sigma}\left(c_{\alpha i}^{\sigma \dagger}\right)$ is the annihilation (creation) operator for an electron at the atomic site $i$ in an orbital $\alpha$ with a spin $\sigma . h$ vanishes in the non-magnetic metal, and $\gamma_{i j}^{\alpha \beta}$ is zero if $i$ and $j$ do not correspond to nearest neighbor sites. Hybridization between the $s$ and $d$ orbitals is taken into account by the non-vanishing term $\gamma^{s d}$. We have chosen to consider two orbitals per site in order to give an appropriate description of the density of states of transition metals and to take into account interband scattering occurring at interfaces between different materials. The DOS of a transition metal consists of a narrow band (mainly $d$-like) embedded in a broader band (mainly $s p$-like). This feature can be reproduced in the above two band model, as shown in reference [16], where the appropriate choices for $\gamma_{i j}^{\alpha \beta}$ and $\epsilon_{i}^{\alpha}$ in $\mathrm{Cu}$ and $\mathrm{Co}$ are discussed.

We analyze the simplest generic model of disorder, introduced by Anderson within the framework of the localization theory [17, which consists of adding a random potential $V_{i}$ to each on-site energy, with a uniform distribution of width $W(-W / 2 \leq V \leq W / 2)$, centered on $V=0$

$$
\tilde{\epsilon}_{i}^{\alpha \sigma}=\epsilon_{i}^{\alpha \sigma}+V
$$

The conductances and GMR ratios are calculated within the Landauer-Büttiker theory of transport [18] using a technique already presented elsewhere [19]. In figure 2 we present the mean GMR ratio for type I (type II) multilayers $\mathrm{GMR}_{\mathrm{I}}\left(\mathrm{GMR}_{\mathrm{II}}\right)$ and the difference between the GMR ratios of type I and type II multilayers $\Delta \mathrm{GMR}=\mathrm{GMR}_{\mathrm{I}}-\mathrm{GMR}_{\mathrm{II}}$, as a function of $\mu$ for different values of the on-site random potential. The average has been taken over 10 different random configurations except for very strong disorder where we have considered 60 random configurations. In the figure we display the standard deviation of the mean only for $\Delta$ GMR because for $\mathrm{GMR}_{\mathrm{I}}$ and $\mathrm{GMR}_{\mathrm{II}}$ it is negligible on the scale of the symbols. It is clear that type I multilayers possess a larger GMR ratio than type II multilayers, and that both the GMR ratios and their difference increase for large $\mu$. These features are in agreement with experiments 13, 14 and cannot be explained within the standard Boltzmann description of transport. The increase of the GMR ratio as a function of the number of bilayers is a consequence of enhancement of the spin asymmetry of the current due to disorder. In fact, despite the Anderson potential being spin-independent it will be more effective on the $d$ band than on the $s$ band, because the former possesses a smaller bandwidth. Since the minority spin sub-band is dominated by the $d$-electrons and the majority by the $s$-electrons, the disorder will suppress the conductance more strongly in the minority band than in the majority. Moreover, since the transport is phasecoherent, the asymmetry builds up with the length, resulting in a length-dependent increase of the GMR ratio. The different GMR ratios of type I and type II multilayers can be understood by considering the inter-band scattering. Both multilayers possess the same conductance in the $\mathrm{P}$ alignment, while the conductance of type 
I multilayer in the AP alignment is smaller than that of type II. The inter-band scattering is very strong when an electron crosses phase-coherently a region where the magnetizations have opposite orientations, and this occurs in each $\left(\mathrm{N} / \mathrm{M} / \mathrm{N} / \mathrm{M}^{\prime}\right)$ cell for type I multilayers, while only in the central cell for type II multilayer (see figure 11a and 1 b). Hence the contribution to the resistance in the AP alignment due to inter-band scattering is larger in type I than in type II multilayers. Finally when the elastic mean free path is comparable with a single $\mathrm{Co} / \mathrm{Cu}$ cell one expects the resistor model to become valid. To illustrate this feature, figure 2 shows that in the case of very large disorder $(W=1.5 \mathrm{eV}), \Delta \mathrm{GMR}$ vanishes within a standard deviation as predicted by the Valert and Fert theory.

As a second example in which the dependence of the GMR ratio on disorder changes when the multilayer geometry is varied, consider the system whose AP alignment is sketched in figure 1 $1 \mathrm{c}$ and 1 $\mathrm{d}$. In this case $\mathrm{M}$ and $\mathrm{M}^{\prime}$ are different materials chosen in such a way that the minority (majority) band of $\mathrm{M}$ possesses a good alignment with the majority (minority) band of $\mathrm{M}^{\prime}$. Moreover the thickness of the $\mathrm{N}$ layers has been chosen in order to allow an AP alignment of the magnetizations of adjacent magnetic layers in both type I and type II multilayers. In this case both type I and type II multilayers exhibit conventional $\mathrm{P}$ and $\mathrm{AP}$ alignments, but their potential profile is quite different. In figure 3 we present a schematic view of the potential profiles for type I and type II multilayers for both the spins in the $\mathrm{P}$ and $\mathrm{AP}$ configuration. A high barrier corresponds to large scattering and a small barrier corresponds to weak scattering. The dashed line represents the effective potential for material $\mathrm{M}$ and and the continuous line for material $\mathrm{M}^{\prime}$. Figure 3 illustrates that type I multilayers possess a high transmission spinchannel in the AP alignment, and hence the resulting GMR ratio will be negative. In contrast type II multilayers do not possess a high transmission channel (there are large barriers for all spins in both the $\mathrm{P}$ and $\mathrm{AP}$ configuration) and the sign of the GMR ratio will depend on details of the band structure of $\mathrm{M}$ and $\mathrm{M}^{\prime}$. Consider the effects of disorder on these two kinds of multilayers. Using the same heuristic arguments as above we expect that the GMR ratio of type I multilayers will increase (become more negative) as disorder increases, in the case of disorder that changes the spin asymmetry of the current. This is a consequence of the fact that, in common with the conventional single-magnetic element, one of the spin sub-bands in the AP alignment is dominated by weak $s$-electrons (small barrier), which are only weakly affected by disorder. It is clear that this system is entirely equivalent to conventional single-magnetic element multilayers discussed above. In contrast for type II multilayers there are no spin sub-bands entirely dominated by the weak scattering (small barriers) $s$-electrons, and all spins in either the $\mathrm{P}$ and $\mathrm{AP}$ configuration will undergo scattering by the same number of high barriers. In this case the effect of disorder will be to increase all the resistances and this will result in a suppression of GMR. Moreover it is important to note that in the completely diffusive regime, where the resistances of the different materials may be added in series, the GMR ratio will vanish if $R_{\mathrm{M}}^{\uparrow(\downarrow)} \sim R_{\mathrm{M}^{\prime}}^{\downarrow(\uparrow)}$, where $R_{\mathrm{A}}^{\uparrow(\downarrow)}$ is the spin-dependent resistance of the material A. To verify this prediction we have simulated both type I and type II multilayers using the parameters corresponding to $\mathrm{Co}$ and $\mathrm{Fe}_{72} \mathrm{~V}_{28}$ of reference [16], respectively for $\mathrm{M}$ and $\mathrm{M}^{\prime}$, and corresponding to $\mathrm{Cu}$ for $\mathrm{N}$. This choice was motivated by the fact that a reverse CPP-GMR has been obtained for $\left(\mathrm{Fe}_{72} \mathrm{~V}_{28} / \mathrm{Cu} / \mathrm{Co} / \mathrm{Cu}\right) \times \mu$ multilayers [20]. The GMR ratio for type I and type II multilayers is shown in figure 4 , which illustrates the remarkable result that the GMR ratio of type I multilayers increases with disorder, while for type II structures it decreases. As explained above this is due to an enhanced asymmetry between the conductances in the $\mathrm{P}$ and AP alignment for type I multilayers, and to a global increase of all the resistances for type II multilayers. As far as we know there are no experimental studies of the consequences of the geometry-dependent effect described above, and further investigation will be of interest, in order to clarify the rôle of the disorder in magnetic multilayers.

Despite the fact that GMR was discovered more than ten years ago, it continues to present fascinating insights into transport in magnetic heterostructures. In this Letter we have addressed a new issue which lies outside the widely-adopted Boltzmann description of GMR, namely that changing the order of magnetic multilayers can significantly alter the magnetoresistance [13, 14. We have shown that this effect is a consequence of phase coherence on a length scale greater than the layer thicknesses.

Acknowledgments: The authors want to thank D.Bozec, C. Marrows, B.Hickey and M.Howson from the University of Leeds for their suggestions and for the permission to discuss results not yet published. This work is supported by the EPSRC, the EU TMR Programme and the DERA.

[1] M.N.Baibich, J.M.Broto, A.Fert, F.Nguyen Van Dau, F.Petroff, P.Etienne, G.Creuzet, A.Friederich, and J.Chazelas, Phys. Rev. Lett. 61, 2472 (1988);

[2] G.Binasch, P.Grünberg, F.Sauerbach and W.Zinn, Phys. Rev. B 39, 4828 (1989)

[3] W.P.Pratt Jr., S.-F.Lee, J.M.Slaughter, R.Loloee, P.A.Schroeder, J.Bass, Phys. Rev. Lett. 66, 3060 (1991)

[4] M.A.M.Gijs, S.K.J.Lenczowski, J.B.Giesbers, Phys. Rev. 
Lett. 70, 3343 (1993)

[5] W.Schwarzacher, D.S.Lashmore, IEEE Trans. Magn. 32, 3133 (1996)

[6] A.Blondel, J.P.Meier, B.Doudin, J.Ph.Ansermet, Appl. Phys. Lett. 65, 3019 (1994)

[7] L.Piraux, S.Dubois, A.Fert, L.Belliard, Eur. Phys. J. B 4, 413 (1998)

[8] M.A.M.Gijs, G.E.W.Bauer, Adv. Phys., 46, 285 (1997)

[9] J-Ph. Ansermet, J.Phys.: Cond. Matter C 10, 6027 (1998)

[10] T.Valet, A. Fert, Phys. Rev. B 48, 7099 (1993)

[11] A. Fert, J.-L. Duvail, T.Valet, Phys.Rev. B 52, 6513 (1995)

[12] S.-F.Lee, W.P.Pratt Jr., R.Loloee, P.A.Schroeder, J.Bass, Phys. Rev. B 46, 548 (1992)

[13] D. Bozec, C. Marrows, B. Hickey, M. Howson, private communication

[14] W.-C. Chiang, Q. Yang, W. P. Pratt Jr., R. Loloee, J. Bass, J. Appl. Phys. 81, 4570 (1997)

[15] C. Marrows, $\mathrm{PhD}$ dissertation, University of Leeds, (1997)

[16] S.Sanvito, C.J.Lambert, J.H.Jefferson, preprint

[17] P.W.Anderson, D.J.Thouless, E. Abrahams, D.S.Fisher, Phys.Rev. B 22, 3519 (1980)

[18] M.Büttiker, Y.Imry, R.Landauer, and S.Pinhas, Phys. Rev. B 31, 6207 (1985)

[19] S.Sanvito, C.J.Lambert, J.H.Jefferson, and A.M.Bratkovsky, accepted for publication in Phys.Rev. B, also cond-mat/9808282

[20] S.Y. Hsu, A. Barthélémy, P. Holody, R. Loloee, P.A. Schroeder, A. Fert, Phys. Rev. Lett. 78, 2652 (1997)

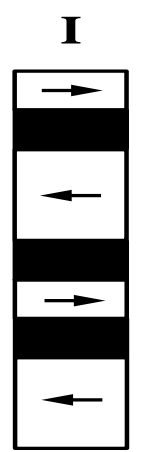

(a)

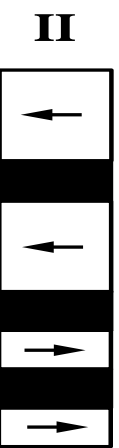

(b)

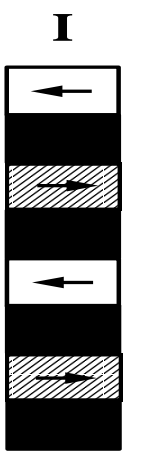

(c)

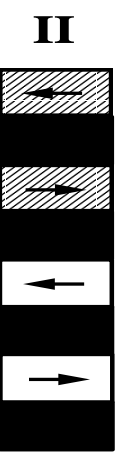

(d)
FIG. 1. AP configuration for type I and type II multilayers described in the text. Figures (a) and (b) refer to the cases of thick and thin Co layers with different coercive fields. Figures (c) and (d) refer to different magnetic metals coupled through non-magnetic spacers via exchange coupling. The black blocks represent $\mathrm{Cu}$, the white $\mathrm{Co}$ and the hatched $\mathrm{Fe}_{72} \mathrm{~V}_{28}$. The arrows indicate the direction of the magnetizations. Note the difference in the case of figure (b) where the AP alignment occurs between the two halves of the multilayer.

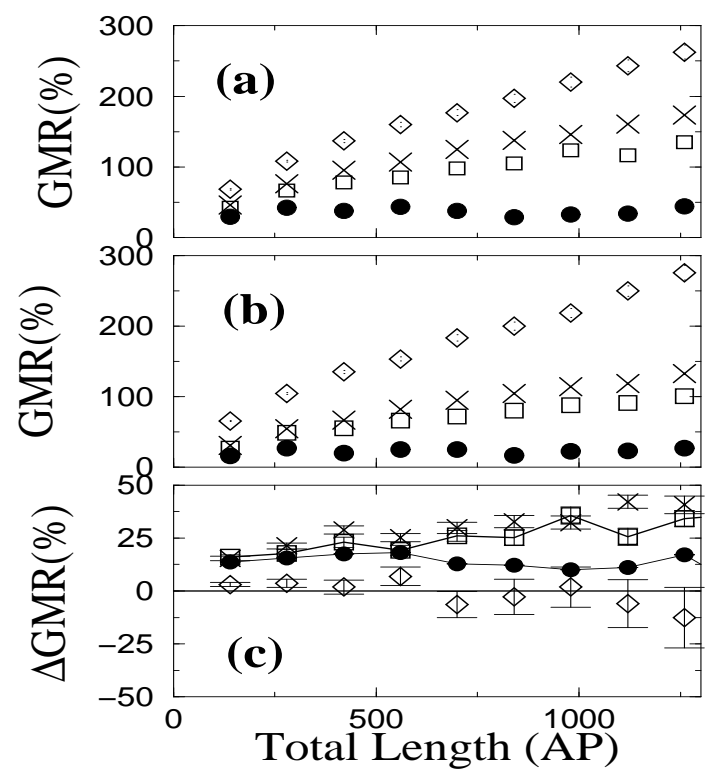

FIG. 2. GMR for type I (a) and type II (b) multilayers, and $\triangle$ GMR (c) in the case of thin (10AP) and thick (40AP) Co layers, as a function of the number of double bilayers $\mathrm{Co} / \mathrm{Cu} / \mathrm{Co} / \mathrm{Cu}$ for different values of disorder. The symbols represent respectively $W=0(\bullet), W=0.3 \mathrm{eV}(\square), W=0.6 \mathrm{eV}$ $(\times), W=1.5 \mathrm{eV}(\diamond)$.
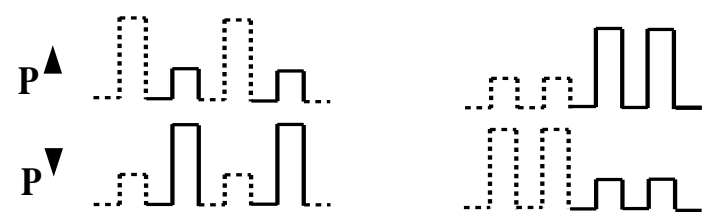

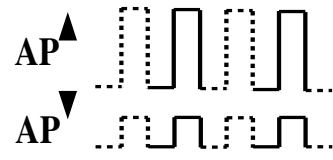

(a)

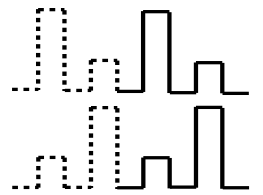

(b)
FIG. 3. Heuristic scattering profiles for type I (a) and type II (b) multilayers of the second example discussed in the text. The dashed and continuous lines represent respectively scattering potentials of material $\mathrm{M}$ and $\mathrm{M}^{\prime}$.

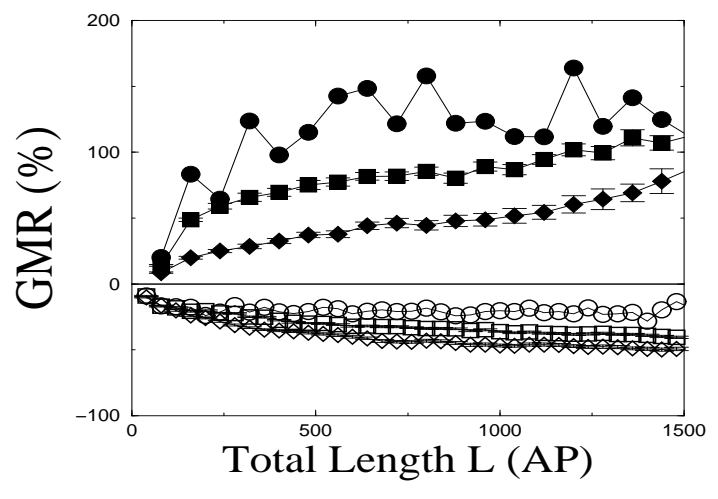


FIG. 4. Different geometry-induced behavior of the GMR ratio as a function of disorder in multilayers composed of Co and $\mathrm{Fe}_{72} \mathrm{~V}_{28}$. In this case all the layer thicknesses are fixed at 10AP. The open (closed) symbols represent type I (type II) multilayers discussed in the text. The circles are the disorder free case, squares and diamonds are for random on-site potentials of $0.6 \mathrm{eV}$ and $1.2 \mathrm{eV}$ respectively. 